\title{
Development of Portable Monitoring System for Real-Time Detection of Fetal Movement
}

\author{
Qifeng Jiang1*, Bin $\mathrm{Gao}^{2}$, Yang $\mathrm{Li}^{2}$, \\ ${ }^{1}$ Department of Biochemistry and Molecular Biology, Chongqing Medical University, Chongqing, China \\ ${ }^{2}$ Department of Medical Information, Chongqing Medical University, Chongqing, China \\ Email: *jqf1008@aliyun.com
}

How to cite this paper: Jiang, Q.F., Gao, B. and Li, Y. (2018) Development of Portable Monitoring System for Real-Time Detection of Fetal Movement. Advances in Bioscience and Biotechnology, 9, 380-389. https://doi.org/10.4236/abb.2018.98026

Received: July 31, 2018

Accepted: August 17, 2018

Published: August 20, 2018

Copyright (c) 2018 by authors and Scientific Research Publishing Inc. This work is licensed under the Creative Commons Attribution International License (CC BY 4.0).

http://creativecommons.org/licenses/by/4.0/

(c) (i) Open Access

\begin{abstract}
Effective fetal monitoring is an important guarantee for fetal health and early treatment. Fetal movement is one of critical indicators of fetal monitoring, which plays an important role in fetal health. Counting the number of fetal movement by pregnant women is a traditional method for long-term monitoring. However, there are many defects in pregnant women's feeling count, which cannot meet the accurate requirements of modern perinatal medicine. With the rapid development of biological and electronic technology, various sensors are used to probe the fetal dynamic monitoring, but not on fetal movement. This research proposes a monitoring method for fetal movement via three electrodes. Briefly: first, three electrodes are used to extract electrical signals in the abdomen of pregnant women; second, these signals are amplified and filtered; third, A/D converter with microprocessor is used to make analog digital conversion, which can be stored in the SD card under the control of the microprocessor; finally, the SD card data are processed by computer software and the fetal movement information is analyzed.
\end{abstract}

\section{Keywords}

Fetal Movement, Monitoring, Electrocardiogram Electrode

\section{Introduction}

Fetal health is highly concerned by family and society. Effective fetal monitoring is an important guarantee for improving fetal health and early treatment [1]. Clinical data show that abnormal umbilical cord and oligohydramnios lead to fetal distress and neonatal hypoxia are the main causes of perinatal fetal death, accompanied by abnormal fetal movement [2] [3]. Fetal movement is a critical indicator of fetal monitoring which plays an important role in fetal health. Pre- 
natal fetal movement monitoring is a traditional screening examination in perinatal period which could indicate fetal intrauterine risk factors before ultrasound examination [4]. Long-term monitoring for fetal movement has an important clinical significance for pregnant women, especially pregnant women with pregnancy induced hypertension [5]. Because of the complexity of abdominal signals and the weakness of fetal movement signals, long-term real-time monitoring of fetal movement has always been a difficult problem in fetal monitoring.

At present, the traditional methods for fetal movement monitoring mainly compose of number counting by pregnant women and hospital no load test (NST). Because of the subjective defects, there is a large error in the method of number counting, which has no significant effect on reducing the mortality of the fetus. With the rapid development of biological and electronic technology, many researches are turned to the motion sensors, for instance the Doppler ultrasound probe. NST with Doppler ultrasonic becomes an important means of perinatal monitoring [6] [7]. However, there are still some defects in Doppler ultrasound for monitoring fetal movement. First, the most common use of Doppler ultrasound at present is regular hospital examination. The method is designed to predict a week of 20 minute fetal movement and fetal heart monitoring which is prone to false positive; second, this test requires repeated ultrasonic monitoring of pregnant women for accuracy, which might induce subjects with conflict of emotion; Most important, the long-term use of Doppler ultrasound is controversial on whether the fetus may get hurt, which could be a damage to the health of fetus [8] [9].

At present, the method of counting monitoring for pregnant women is obviously unable to meet the needs of modern perinatal medicine. Although the Doppler ultrasound monitoring and fetal movement used in hospital is relatively mature, it cannot be used for long-term monitoring of domestic fetal movement monitoring. This study proposes a monitoring method for fetal movement via three electrodes. First, three electrodes are used to extract electrical signals in the abdomen of pregnant women; Second, these signals are amplified and filtered; Third, A/D converter with microprocessor is used to make analog digital conversion, which can be stored in the SD card under the control of the microprocessor; Finally, the SD card data is processed by computer software and the fetal movement information is analyzed.

\section{System Construction}

\subsection{Hardware}

The overall design of the instrument hardware is shown in Figure 1. The instrument is mainly composed of the ECG electrode, the analog signal processing part and the digital signal processing section, in which ECG electrodes are uses for signal collection, and analog signal processing part is mainly used to amplify the signal and filter the high frequency plus direct current interference. The digital signal processing part mainly controls the modules and processing and 


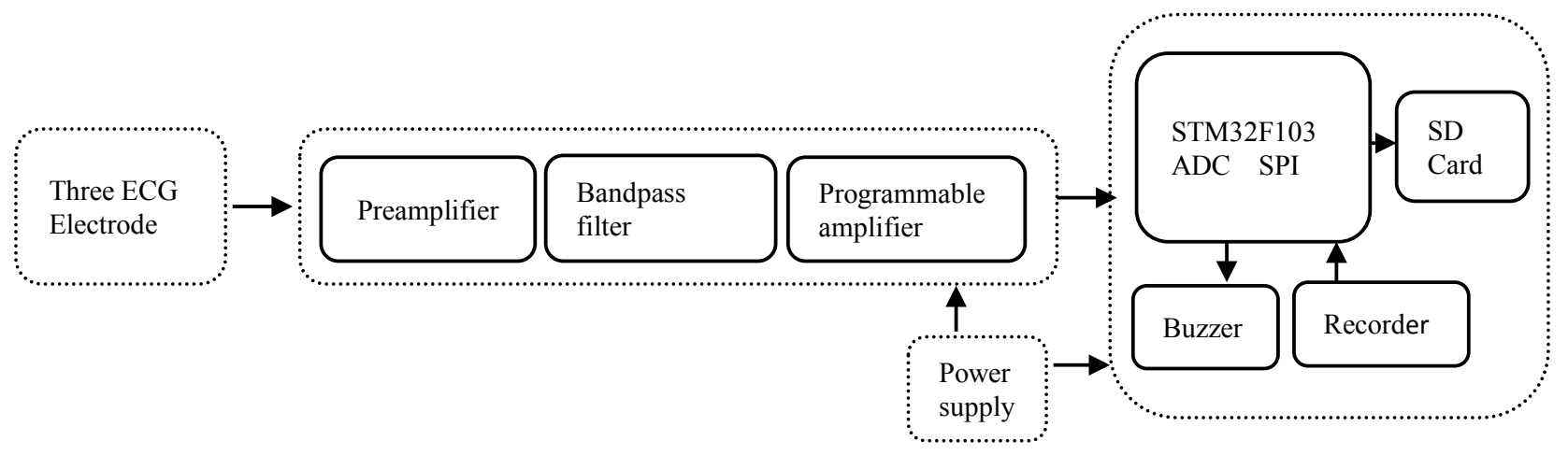

Figure 1. Structure block diagram of instrument.

storing the acquisition signals. The power supply unit of the instrument was composed of rechargeable lithium battery and conversion power supply module, which provides the required voltage for each module of the instrument with continuous power supply. Besides, convenient SD card was used as data storage for further analysis.

\subsubsection{Preamplifier}

The input of the preamplifier circuit is the ECG electrode and the output is band-pass filter. The ideal effect is that the signal collected by the ECG electrode is fully received and output to the band pass filter, and no external electromagnetic interference signal is received, and the signal is magnified at the same time. As shown in Figure 2, in order to approach the ideal effect, the preamplifier designed in this study can be divided into two parts. The first part is a passive low pass filter and a limiting circuit, and the second part is a preamplifier. The AD4 chip uses LMC6082 integrated block, and its internal consists of two integrated amplifiers. The first integrated amplifier is used to build a voltage follower in which the acquisition signal of the control electrode could meet the requirements for the simultaneous control voltage for multiple working electrodes (input). Second integrated amplifiers are also used to build a voltage follower that connects the back electrode (FMGND) to the analog (AGND) circuit of the circuit to meet the needs of the human body and the circuit. The preamplifier output is followed by a low pass filter whose cut-off frequency is about $1.6 \mathrm{KHz}$.

\subsubsection{Bandpass Filter}

The bandpass filter is shown in Figure 3. The main chip is LMC6082 containing two integrated amplifiers. The circuit consists of a second-order high pass filter circuit and a second-order low pass filter circuit. The principle is the output of the second-order high pass filter circuit is output according to the high input impedance and low output impedance of the second-order high pass filter. For the input signal of the second-order low pass filter circuit, bandpass filtering is realized. AV1.5V is the bias voltage, raising the signal baseline from $0 \mathrm{~V}$ to $1.5 \mathrm{~V}$, so that the signal will not distort after entering the digital signal processing circuit. 


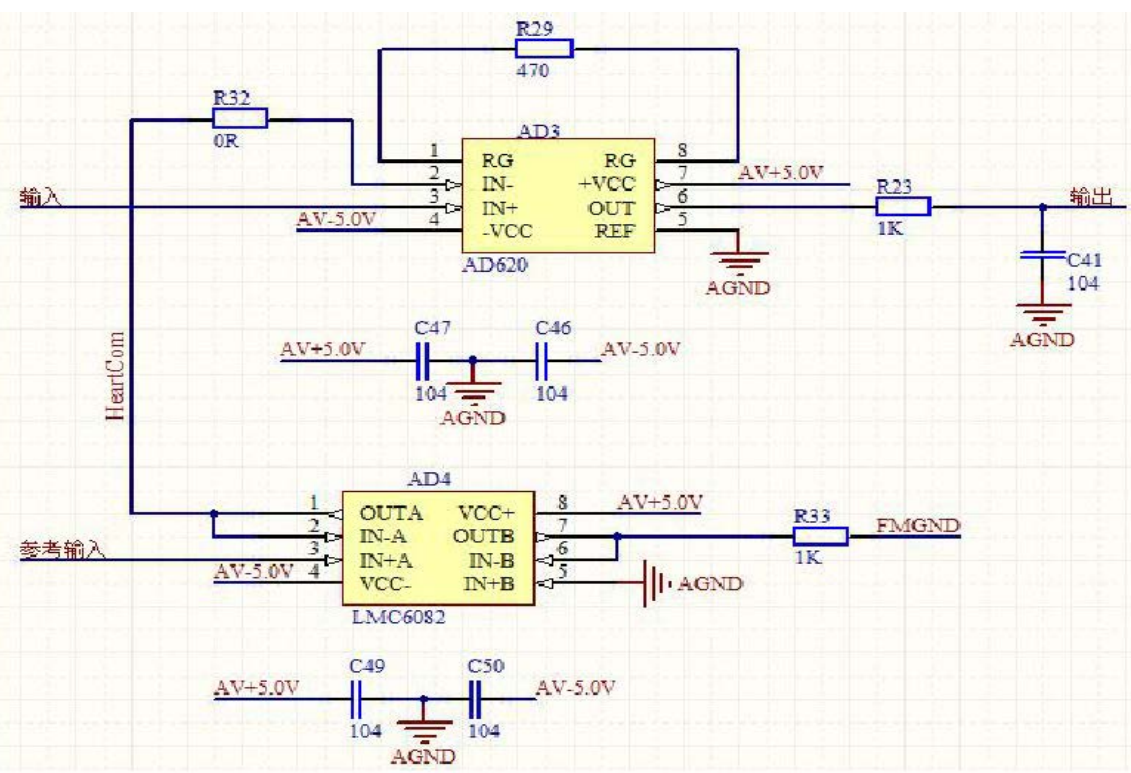

Figure 2. The construction of preamplifier.

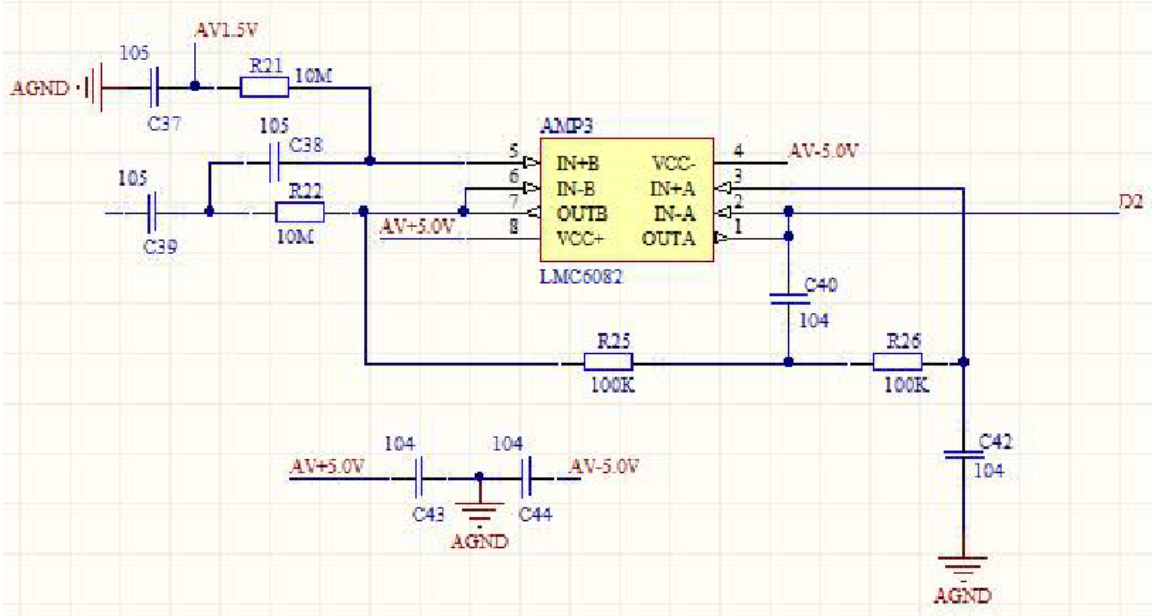

Figure 3. Circuit of bandpass filter.

\subsubsection{Programmable Amplifier}

The programmable amplifier is mainly composed with PGA116 integrated block to construct the circuit diagram as shown in Figure 4, in which the circuit is integrated. In PGA116 module, the circuit diagram is simpler. The output is connected to the input of the ADC module of the microprocessor. The magnification can be changed by sending control commands through the SPI module of the microprocessor.

\subsubsection{Digital Signal Processing Circuit}

The design of digital signal processing circuit is mainly based on the STM32F103 microprocessor, which could meet the requirements of long term fetal movement monitoring port, high sampling frequency and large amount of data. The main function of STM32F103 is to control each unit module to calculate, process 


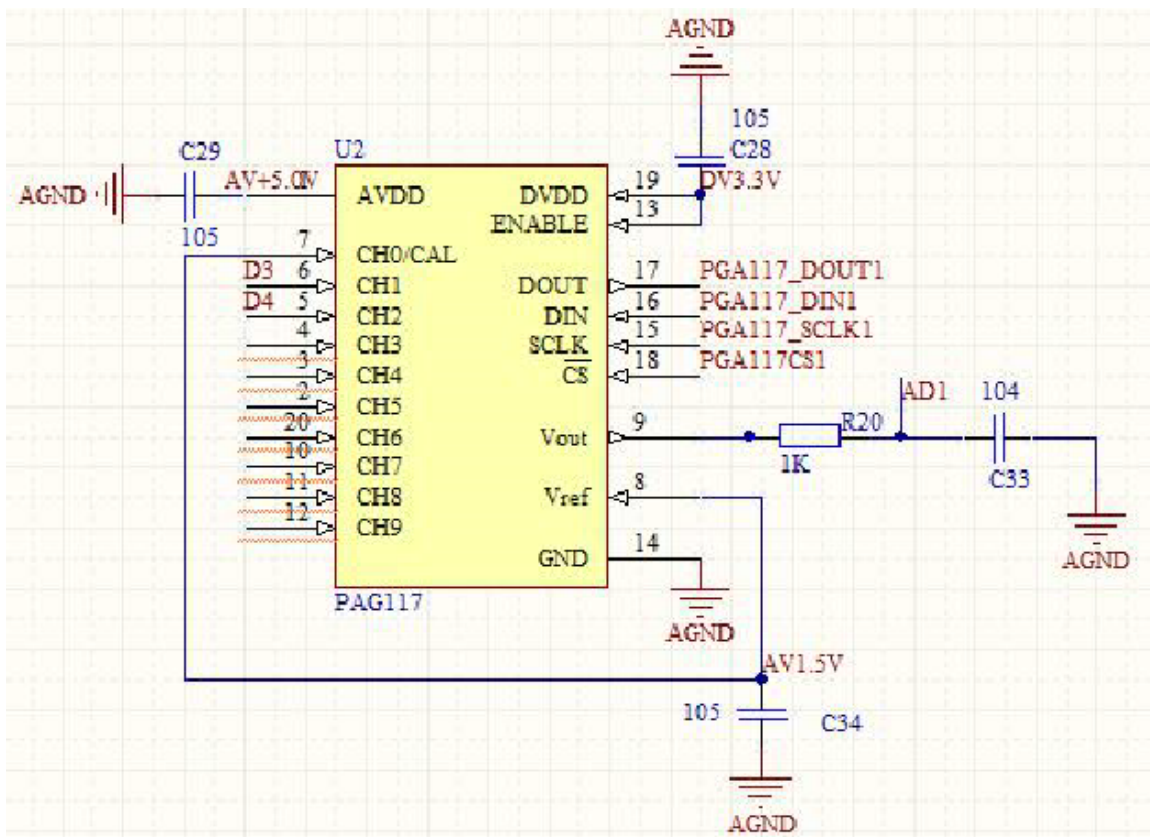

Figure 4. Circuit of programmable amplifier.

and temporarily store data. The interface circuit is shown in Figure 5. The STM32F103 is supplied by analog power supply of $3.3 \mathrm{~V}$ and digital power supply of $3.3 \mathrm{~V}$. The microcontroller connects $8 \mathrm{M}$ crystal oscillator, NRST connection reset circuit, ISP1, BOOT0 and BOOT1 for program burning, PA2 is $\mathrm{ADC}$ interface, and AV1.5 V analog power supply is used to calibrate acquisition signal. Other interfaces are used to control each functional module.

\subsection{Processing Software Design}

The software design of fetal movement monitoring is to convert the collected signal into digital signal and store it in SD card for computer software processing. The data acquisition program involves the configuration of PGA, ADC, the interrupt and the ADC_SPI function. The basic idea of this design is that the configuration of PGA needs to change the magnification and channel two parameters, while the configuration of ADC is continuously sampled, the sampling frequency and channel change are completed by timing interruption, interrupting each time, reading the results of sampling and changing the channel. The PGA configuration and ADC_SPI function program are described in detail below. Besides, the data storage program writes the data into the SD card by the buffer register. The buffer register is the result of the ADC sampling in the acquisition program. The data is stored alternately with two registers. When the pointer of a register points to the last byte of the register, the data of the register is stored and the other register is in the register.

\section{Results and Clinical Application}

There was no ethical conflict in this study, all pregnant women and non-pregnant women were informed before tests which were approved by ethic committee. 


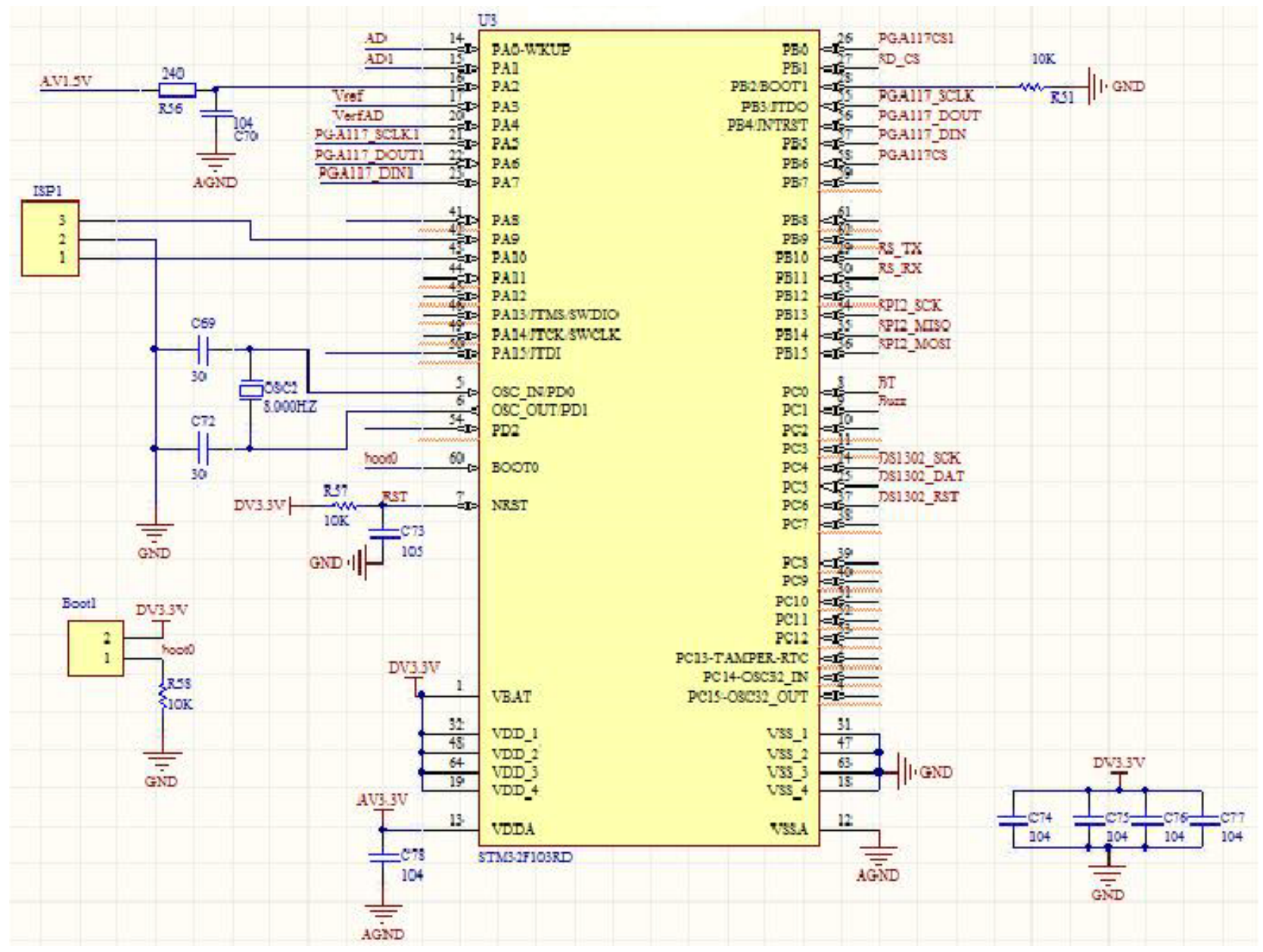

Figure 5. Circuit of digital signal processing.

Basically, 21 healthy pregnant women in Three Gorges Central Hospital of Wanzhou, Chongqing were selected for this test with the average age of 28 . Besides, there were 6 - 8 people in three groups, in terms of 15, 24, 39 weeks of pregnancy which because from $15^{\text {th }}$ week, fetal movement slightly began, which would become frequently at $24^{\text {th }}$ week. When the gestational week reached 39 , fetal movement would decrease but with more strong single movement. The control group had 20 normal females without pregnancy. Both experimental group and control group were informed of the experimental process and were familiar with the operation of the instrument. In the experiment setting, the non-pregnant women in control group were in the same condition as the pregnant women in the experimental group. The abdominal signals were collected at the same time by two experimental devices. Meanwhile, the experimental group took the subjective feelings of pregnant women as a control signal. The whole experiment required the pregnant woman to concentrate on the fetal movement and press the recorder button on the device to record when she felt it. The experiment was divided into two stages. In the first stage, pregnant women and non-pregnant women were in a state of resting or sitting. The second stage ex- 
perimental group and the non-pregnant women were in the daily activities such as walking, limb movement, or trunk movement. The experiment was carried out at very frequent stages of fetal movement, and several stages of fetal movement could be concluded at each stage.

The Figure 6 demonstrated the wave changes during the fluctuation of the signal in the control group and the experimental group with and without fetal movement in the daily activity state. Figure 6(a) was the only waveform in control group, which was characterized by the coincidence of upper electrode and lower electrode with an upward pulse and normal amplitude. As shown in Figure 6(b), when the pregnant women were recorded as the fetal movement, the waveform showed fluctuating differences, in which the upper electrode was obviously larger than the lower electrode in the map. Figure 6(c) was the waveform when the pregnant women did not show fetal movement in their daily activities and the data collected by the two electrodes coincided and remained stable.
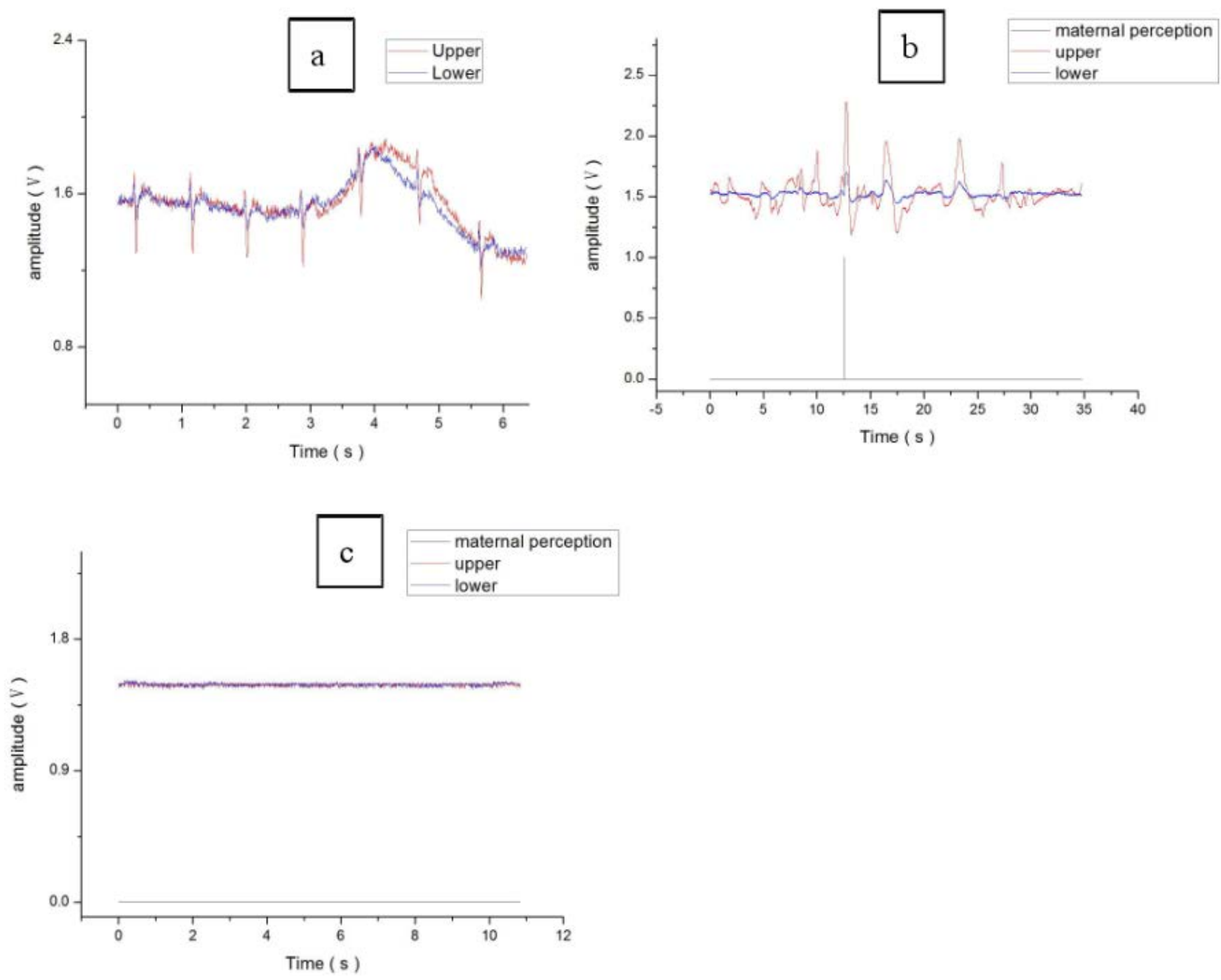

Figure 6. The waveform of experimental and control group in different stage. a: the waveform of control group; (b) the waveform of experimental group with fetal movement; (c) the waveform of experimental group without fetal movement. 
Furthermore, we compared the fetal movement signals between pregnant women with different gestational weeks. As shown in Figure 7, Figure 7(a) was the acquisition signal and sensory signal for pregnant women of 39 weeks of pregnancy, and 12 suspicious fetal movements appeared in the collected signals, of which 11 were felt by pregnant women and recorded as fetal movement; Figure 7(b) for 24 weeks pregnant women's acquisition signal and the sensory signal, the signal in the acquisition of 6 suspicious fetal motion waveform, 4 of them were pregnant women felt and recorded as fetal movement; Figure 7 (c) for 15 weeks pregnant women's acquisition signal without the actual feeling of subjects.

\section{Discussion and Conclusion}

Fetal movement monitoring is charactered as the long-term, real-time, accurate and convenient carrying, based on this, a new type of fetal dynamic monitor is proposed in this study according to the present research situation. This system used three electrodes to form signals acquisition part. The hardware circuit and
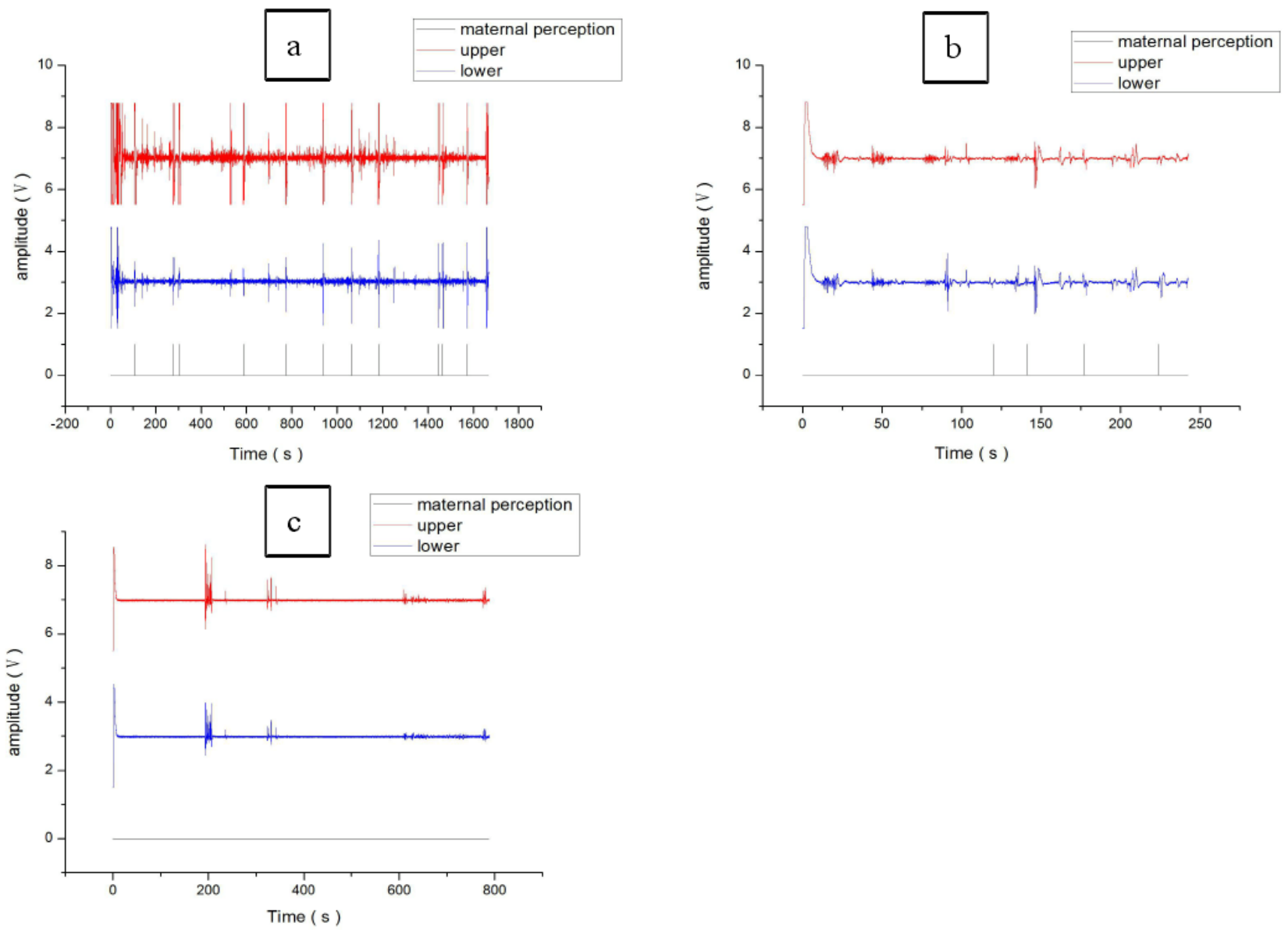

Figure 7. Fetal movement signals of different gestational weeks. The significant fetal movement signals were monitored at 15 weeks of pregnancy. With the increase of gestational weeks, the fetal movement signals increased gradually under the same experimental conditions, and the 39 weeks of waveform showed the most frequent fetal movement signals. (a) 39 gestational weeks fetal movement signal; (b) 24 gestational weeks fetal movement signal; (c) 15 gestational weeks fetal movement signal. 
software program were designed to transmit the signal into computer. The performance test and preliminary analysis of the device showed that it could monitor fetal movement signals much more accurate than number counting of pregnant women. Besides, it would eliminate the external interference caused by maternal movement and environment. In addition, this system not only recorded fetal movement signals, but also contained important information such as intensity and time distribution of fetal movement. More important, this system had the advantages of simple operation, high sensitivity, no harm and discomfort, and could be applied to the long-term monitoring at home.

It's worth noting that the waveform of the pregnant women in the experimental group was mainly composed of three waveforms in Figure 6(b), while the non-pregnant women in the reference group had only two waveforms such as Figure 6(a). This waveform would appear in the experimental group of the pregnant women at resting state without fetal movement record, it might be interpreted as other physiological signals. In addition, significant maternal ECG signals could be collected in the control group compared with experimental group, because the control group amplification was much larger than the experimental group. The whole waveform of Figure 6(b) lasted for 34 seconds, indicating that the fetus was moving for about 34 seconds, which was also different from the time that the Figure 6(a) waves fluctuated only a few seconds, which indicated this system not only recorded fetal movement signals, but also contained intensity and time distribution of fetal movement. Figure 6(c) showed that daily movement had little or no effect on data collection by electrodes. Other waveforms in experimental group, which were not recorded as fetal movements by the pregnant women and did not appear in the signals collected in control group, might be suspicious of fetuses. Whether the characteristic of dynamic waveform is fetal movement needs further study. The daily activities such as body movement and trunk movement of subjects showed no significant influence on the data acquisition, and the three electrodes array could also be well recognized other physiological signals and suspected fetal motion signals. Therefore, it can be explained that the system could collect certain fetal movement signals with good elimination on interference. The limitation of this system could be interpreted that the manual counting of the peak is needed by now and the test results would be compared with ultrasonic measurement in the future.

\section{Acknowledgements}

This study was supported by NSFC (National Natural Science Foundation of China, No. 31201047 to Jiang), by SRFDP (Research Fund for the Doctoral Program of Higher Education, No. 20125503120018 to Jiang), by the open fund of Key Laboratory of Ministry of Education (No. CQKLBST-2012-005 to Jiang), by the Chongqing Yuzhong District Science and technology project (No. 20150115) 


\section{Conflicts of Interest}

The authors declare no conflicts of interest regarding the publication of this paper.

\section{References}

[1] Manning, F.A., et al. (1980) Antepartum Fetal Evaluation: Development of a Fetal Biophysical Profile. American Journal of Obstetrics \& Gynecology, 136, 787-795. https://doi.org/10.1016/0002-9378(80)90457-3

[2] Moore, T.R. and Piacquadio, K. (1989) A Prospective Evaluation of Fetal Movement screening to Reduce the Incidence of Antepartum Fetal Death. American Journal of Obstetrics \& Gynecology, 160, 1075-1085.

https://doi.org/10.1016/0002-9378(89)90164-6

[3] Mangesi, L. and Hofmeyr, G.J. (2007) Fetal Movement Counting for Assessment of Fetal Well-Being. DB SYST REV, 9, 203-206.

[4] Fr en, J.F. (2004) A Kick from Within-Fetal Movement Counting and the Cancelled Progress in Antenatal Care. Journal of Perinatal Medicine, 32, 13-24.

[5] Reddy, U.M. (2007) Prediction and Prevention of Recurrent Stillbirth. Obstetrics \& Gynecology, 110, 1151-1164. https://doi.org/10.1097/01.AOG.0000287616.71602.d0

[6] Mangesi, L., et al. (2007) Fetal Movement Counting for Assessment of Fetal Wellbeing. The Cochrane Library, New York.

[7] Heazell, A.E.P. and Fren, J.F. (2008) Methods of Fetal Movement Counting and the Detection of Fetal Compromise. American Journal of Obstetrics \& Gynecology, 28, 147-154. https://doi.org/10.1080/01443610801912618

[8] Sheikh, M., et al. (2014) Maternal Perception of Decreased Fetal Movement from Maternal and Fetal Perspectives, a Cohort Study. BMC Pregnancy Childbirth, 14, 1-7. https://doi.org/10.1186/1471-2393-14-286

[9] Fr en, J.F., et al. (2008) Fetal Movement Assessment. Seminars in Perinatology, 32, 243-246. https://doi.org/10.1053/j.semperi.2008.04.004 